\title{
Politique
}

Politique

\section{Les partis politiques municipaux et les élections municipales de 1986 à Montréal et de 1989 à Québec}

\section{Serge Belley}

Numéro 21, hiver 1992

URI : https://id.erudit.org/iderudit/040711ar

DOI : https://doi.org/10.7202/040711ar

Aller au sommaire du numéro

Éditeur(s)

Société québécoise de science politique

ISSN

0711-608X (imprimé)

1918-6584 (numérique)

Découvrir la revue

Citer cet article

Belley, S. (1992). Les partis politiques municipaux et les élections municipales de 1986 à Montréal et de 1989 à Québec. Politique, (21), 5-35.

https://doi.org/10.7202/040711ar

\section{Résumé de l'article}

Les élections de 1986 à Montréal et de 1989 à Québec représentent deux cas importants de changement politique au niveau municipal. Outre la conjoncture politique, trois facteurs sont considérés ici pour rendre compte du renversement des partis " civiques " par les partis " populaires " : le changement de leadership au sein des partis, le contenu de leurs plates-formes électorales et la définition des enjeux électoraux. Malgré un certain rapprochement, l'analyse montre qu'il existe encore des différences idéologiques marquées entre ces deux types de partis. C'est le cas notamment en matière de démocratie et d'aménagement qui ont été, dans les deux villes, les deux principaux enjeux électoraux. 


\section{LES PARTIS POLITIQUES MUNICIPAUX ET LES ÉLECTIONS MUNICIPALES DE 1986 À MONTRÉAL ET DE 1989 À QUÉBEC*}

\section{Serge Belley}

Université Laval

Les élections de 1986 a Montréal et de 1989 a Québec représentent deux cas importants de changement politique au niveau municipal. Outre la conjoncture politique, trois facteurs sont considérés ici pour rendre compte du renversement des partis «civiques» par les partis epopulairesn: le changement de leadership au sein des partis, le contenu de leurs plates-formes électorales et la définition des enjeux électoraux. Malgré un certain rapprochement, l'analyse montre qu'il existe encore des différences idéologiques marquées entre ces deux types de partis. C'est le cas notamment en matière de démocratie et d'aménagement qui ont été, dans les deux villes, les deux principaux enjeux électoraux.

Les élections municipales de 1986 à Montréal et celles de 1989 à Québec marquent un changement important dans la direction politique de ces villes. Ces élections ont abouti à la défaite du Parti civique de Montréal (PCM) et du Progrès civique de Québec (PCQ), deux formations politiques qui exercaient respectivement le pouvoir depuis 1960 et 1965 . Après avoir joué pendant une douzaine d'années le rôle de partis d'opposition, le Rassemblement des citoyens et des citoyennes de Montréal (RCM) et le Rassemblement populaire de Québec (RPQ) accédaient pour la première fois au statut de partis gouvernementaux.

Sur la base de quelles plates-formes électorales ce changement politique s'est-il produit? Les différences idéologiques qui, au début des années 80 , opposaient encore fortement les partis municipaux de la première génération, dits "civiques", et les partis municipaux de la deuxième génération dits "populaires" (Quesnel-Ouellet, 1982), se retrouvent-elles 
dans les plates-formes électorales de 1986 et de 1989 ? En dépit des caractéristiques sociodémographiques et économiques particulières à chacune des deux villes, les campagnes électorales qui s'y sont déroulées en 1986 et en 1989 ont-elles donné lieu à un débat électoral animé par les mêmes enjeux ou thèmes mobilisateurs?

Ces trois questions font l'objet du présent texte. Pour y répondre, nous avons analysé dans la presse écrite le contenu des plates-formes électorales rendues publiques par les différents partis au cours des deux élections qui nous intéressent. L'analyse comparative met en lumière non seulement les différences et les ressemblances entre les deux types de partis («civiques" et «populaires») plusieurs années après leur création, mais également les points qui rapprochent et éloignent les partis de même "famille" politique.

Dans la première partie du texte, plus théorique et méthodologique, il est question d'abord des partis politiques municipaux et de leur environnement. On aborde aussi le leadership et l'impact qu'un changement de chef a pu avoir sur les programmes officiels des partis et sur leurs plates-formes électorales. Puis suivent des précisions sur la façon dont nos données ont été recueillies et classées. La deuxième partie est consacrée à l'analyse des plates-formes électorales lors de l'élection de 1986 a Montréal et de l'élection de 1989 à Québec. Une analyse comparative de ces deux campagnes électorales constitue la troisième partie.

\section{Les partis politiques municipaux et leur environnement}

Tout comme les partis politiques présents aux autres paliers de gouvernement, les partis municipaux, qu'ils aspirent a prendre ou à conserver le pouvcir, doivent tôt ou tard tenir compte des multiples changements qui se produisent tant a l'extérieur qu'à l'intérieur du système politique. Les partis, leurs programmes, leurs décisions (au gouvernement ou dans l'opposition) et, conséquemment, les relations interpartisanes sont sans cesse sujets à transformations. D'une part, ces transformations prennent sources dans les clivages démographiques, ethniques, socio-économiques et idéologiques qui marquent l'environnement sociétal. D'autre 
part, elles résultent des changements apportés aux règles électorales et au fonctionnement des institutions politiques (Lemieux, 1985: 223-225). La naissance même des partis est a mettre en relation avec ces deux sources de changements.

La création des premiers partis politiques municipaux dans les villes de Montréal et de Québec a effectivement été engendrée par l'inadéquation de plus en plus grande entre les politiques des élus locaux («indépendants") et des mutations urbaines profondes.

Ces villes ont connu, en effet, après la Deuxième Guerre mondiale, une croissance démographique rapide, un développement du secteur tertiaire, l'exode vers les banlieues, la dégradation du tissu urbain et des infrastructures, la désuétude des structures politiques et administratives, etc. C'est sur cette base que se sont créés, à Montréal, la Ligue d'action civique en 1954 et le Parti civique de Montréal' en 1960. A Québec, en 1962, ce sera au tour du Progrès civique de Québec.

Ces partis municipaux sont portés au pouvoir grâce à des programmes axés sur des thèmes comme la lutte au patronage et à la corruption, la modernisation administrative, la rénovation urbaine et le progrès économique. Les deux villes adoptent aussitôt des réformes importantes aussi bien de leur systeme politico-administratif que de leur aménagement et de leur développement (Kaplan, 1982: 420-430; Sancton, 1983: 68-72; EZOP-Québec, 1981; Quesnel-Ouellet, 1976).

Au tournant des années 70 , deux conditions ont été propices à l'émergence de partis de type "populaire" dans les villes de Montréal et Québec. L'affirmation des idéologies nationaliste, sociale-démocrate ou plus ouvertement marxiste, portées par une génération plus jeune, plus instruite et associée au développement des secteurs public et parapublic, est l'une de ces conditions. L'autre est la montée concomitante des luttes urbaines et des mouvements

1. Le fondateur du PCM, Jean Drapeau, a été élu pour la première fois à la mairie de Montréal en 1954 sous la bannière de la Ligue d'action civique. La ligue présentera des candidats aux élections municipales jusqu'en 1966. 
d'opposition aux politiques d'aménagement et de développement prônées par les partis civiques. Ainsi naîtront a Montréal, en 1970, le Front d'Action politique (FRAP) et, quatre ans plus tard, le Rassemblement des citoyens et des citoyennes de Montréal (RCM). En 1977, naît le Rassemblement populaire de Québec (RPQ). Cette deuxième génération de partis municipaux prône les mêmes objectifs : démocratie locale, décentralisation politique et administrative, développement des services sociaux et communautaires, priorité au logement public et a la qualité de vie dans les quartiers.

A compter de la fin des années 70 , certaines interventions législatives du gouvernement provincial en matière d'urbanisme, de démocratie locale ${ }^{2}$, de fiscalité et de représentation au sein des communautés urbaines (Quesnel-Ouellet, 1980) auront un impact sur la gestion politique des villes de Montréal et de Québec et, par conséquent, sur leurs partis et leurs systemes partisans. D'autres facteurs entraîneront aussi des transformations dans les partis municipaux et modifieront l'orientation des politiques publiques de ces deux villes au cours de la décennie 80. Au nombre de ces facteurs, on note la récession économique de 1981-1982, la montée des idées néo-libérales prônant la réduction de la taille et du rôle de l'État, et les préoccupations d'une partie croissante de la population envers la protection de l'environnement et du patrimoine en milieu urbain (Andrew, Léveillée et Quesnel, 1985; Léonard et Léveillée, 1986a et 1986b; Léveillée, 1986 et 1988; Quesnel et Belley, 1991).

Si tous ces changements se sont imposés objectivement aussi bien aux partis civiques qu'aux partis populaires, leur influence s'est exprimée encore plus singulièrement sur le plan de leur "leadership".

2. On pense ici à la reconnaissance des partis politiques municipaux, au remodelage de la carte électorale favorisé par l'adoption du principe d'un conseiller par district, au contrôle du financement des partis et des dépenses électorales et a l'octroi d'un budget de recherche aux partis politiques d'opposition dans les villes de Laval, de Montréal et de Québec. 
Leadership et discours électoral : une hypothèse

Les promesses et les actions des autres partis de méme que les changements qui se produisent a l'intérieur et à l'extérieur du système politique peuvent influencer la substance du discours électoral d'un parti au cours d'une campagne électorale. Il est de même des changements qui surviennent au sein même d'un parti : ils peuvent avoir un effet sur son programme et sa plate-forme électorale. Parmi ces modifications internes, celles qui touchent la direction ou le "leadership" du parti ont une importance particulière.

C'est pour imposer son style et affirmer son "leadership" qu'un nouveau chef tente d'apporter au sein du parti certains changements, dont les plus importants concernent généralement le programme (Pelletier, 1989: 108). Celui-ci essaiera d'exercer son influence soit par la suppression de certains articles du programme, soit par l'ajout de nouveaux ou par l'adoption d'un programme officiel si le parti n'en a pas. Le nouveau venu qui est recruté hors des rangs du parti souhaite souvent changer substantiellement le programme; cela peut même être une condition de son engagement. S'ils sont mal acceptés, ces changements pourront être une source de tensions entre les tenants de l'orthodoxie et les réformistes au sein du parti. Tensions qui seront d'autant plus lourdes de conséquences sur le plan électoral que l'installation contestée du nouveau chef précédera de peu la date des élections. S'ils sont bien acceptés, au contraire, l'arrivée du nouveau chef et les changements qu'il veut apporter au programme pourront être non seulement une nouvelle source de cohésion et de dynamisme pour le parti, mais ils pourront devenir des atouts stratégiques en vue de la prochaine campagne électorale (Quesnel et Belley, 1991). Les élections municipales de 1986 à Montréal et celles de 1989 à Québec présentent, de ce point de vue, un intérêt particulier puisque trois des quatre partis en présence (le PCM, le PCO et le RPQ) venaient, dans les mois ayant précédé le déclenchement des élections, de se choisir un nouveau chef hors de leurs rangs.

Mais tout de suite il convient d'établir une distinction entre le programme politique officiel d'un parti et sa 
plate-forme électorale qui regroupe les engagements électoraux rendus publics au cours de la campagne. Le programme officiel serait plus "permanent» et «rigide", tandis que la plate-forme électorale, qui découle logiquement du programme, serait "temporaire» (la durée d'un mandat) et "flexible" (liée aux circonstances particulières qui entourent chaque élection). Cette distinction est tout particulièrement importante du point de vue de l'électeur qui ignore le plus souvent le contenu des programmes officiels des partis. $D^{\prime}$ ailleurs, ceux-ci n'ont pas toujours de programme officiel; ce fut le cas du PCM avant 1986 et du PCQ avant 1989. Le programme, quand il existe, n'est pas toujours facilement accessible, et il arrive que l'électeur $n$ 'ait pas le temps ou le dessein de le consulter (Monière, 1988: 67). C'est pourquoi le contenu du discours électoral diffusé par un parti pendant une campagne a tant d'influence : il oriente pour une grande part l'opinion des électeurs sur les options politiques en présence, et la qualité des candidats et des chefs.

Cependant, en campagne électorale, un parti ne peut pas se contenter de livrer son message. II doit aussi prouver aux électeurs, dans le cadre d'un débat forcément polémique et fortement médiatisé, que ses positions sont dans les circonstances supérieures à celles de ses adversaires. L'enjeu du débat électoral consiste donc, pour un parti, «à installer en position dominante sa représentation de la situation politique» (Gerstlé, 1992: 69). Par conséquent, pour que le changement politique se produise, il ne suffit pas au parti aspirant de tenir compte dans son programme de la conjoncture politique; il lui faut aussi, par son discours électoral, parvenir à construire et à imposer ula" réalité politique. Le RCM et le RPO y sont parvenus d'autant mieux en 1986 et en 1989 qu'ils ont réussi - c'est notre hypothèse - à imposer leurs thèmes de marque et à en faire des enjeux électoraux fortement mobilisateurs, cela à la faveur d'un renouvellement difficile du "leadership».

Précisions méthodologiques

Le discours électoral diffusé par un parti au cours d'une campagne contient plusieurs types $d$ 'informations (critiques, énoncés de principes, réalisations passées, présentations de 
soi, etc.) (Monière, 1988: 69). Mais ce sont les propositions de politiques (les vagues comme les spécifiques), c'est-à-dire ce que promet de réaliser le parti s'il est porté au pouvoir, qui retiennent le plus l'attention.

Sur la base de ce principe, nous avons considéré que pour reconstituer les plates-formes électorales des partis, nous devions retenir tous les engagements électoraux rendus publics par les partis au cours des campagnes officielles. Ils sont tirés d'une analyse de contenu de tous les reportages publiés dans Le Devoir, Le Journal de Montréal, The Gazette, et La Presse, pour Montréal, et dans Le Soleil, pour Québec ${ }^{3}$. Les propositions de politiques ont été comptabilisées une seule fois de sorte que le total des engagements correspond à autant d'engagements différents.

Les engagements rendus publics par les partis ont ensuite été classés à l'aide d'une grille qui renvoie aux différents champs d'intervention des municipalités, champs regroupés en quatre grandes missions : gouvernementale et administrative, communautaire, travaux publics et environnement, aménagement et développement. La mission gouvernementale et administrative comprend les champs : relations intergouvernementales, finances, gestion politique et gestion administrative. La mission communautaire recouvre les champs : sécurité publique, santé et services sociaux, sports et loisirs, et culture. Les travaux publics et environnement englobent la voirie, le transport et l'environnement. L'aménagement et le développement incluent l'urbanisme, l'habitation et le développement économique.

\section{La campagne électorale de 1986 à Montréal}

Quelques événements marquants méritent d'être rappelés pour bien faire comprendre le contexte de cette élection et le discours électoral des partis en présence. Mentionnons d'abord qu'après avoir dirigé le Parti civique de Montréal depuis sa création en 1960, le maire Jean Drapeau

3. La couverture de la campagne faite par Le Journal de Québec nous étant apparue moins complète et précise, ce journal n'a pas été retenu. 
annonce, au printemps 1986, qu'il quitte la direction du parti et ne sera pas candidat aux prochaines élections. Ayant presque toujours compté sur la popularité personnelle de leur chef pour assurer leur propre rélection, les membres élus du parti sont obligés de dénicher un candidat prestigieux pour combler le départ de Jean Drapeau. L'opération s'avère plus difficile que prévu. Deux aspirants candidats, Yvon Lamarre, président du comité exécutif, et Pierre Lorange, coordonnateur du parti, voient leur candidature refusée par Drapeau lui-même (Leblanc, 1989). Le maire sortant leur préfère Roger $D$. Landry, président-éditeur de La Presse, qui, après réflexion, décline l'invitation (Bernard, 1990). C'est finalement un illustre inconnu sur la scène politique municipale, Claude Dupras, ingénieur et homme d'affaires, qui est choisi chef du parti. Élu par le caucus parmi quatre candidats, Dupras accède à la direction du parti en juillet 1986, soit moins de quatre mois avant les élections.

De son côté, le RCM, pour la première fois depuis sa création en 1974, envisage réellement la prise du pouvoir. L'une des raisons de cet espoir est le fait que son chef, Jean Doré, qui en est à sa deuxième tentative à la mairie, a réussi à se faire élire conseiller municipal en mai 1984, à la suite d'une élection partielle. Jean Doré a donc eu deux ans et demi pour se faire connaître à titre de chef de l'opposition officielle, ce qui lui donne un avantage certain sur son adversaire, nouveau venu sur la scène politique municipale. En outre, le RCM n'a pas cette fois à affronter un troisième parti, le Groupe d'action municipale (GAM), dont la présence aux élections municipales de 1978 et 1982 avait profité au PCM ICollin, 1986; Beauregard, 1986; Gagnon-Lacasse et Bourassa, 1986).

Les espoirs des supporters et candidats du RCM s'avéreront fondés : le RCM remporte une éclatante victoire. Jean Doré est élu à la mairie avec plus de $68 \%$ des suffrages. Aux postes de conseillers, le RCM, avec $63 \%$ des voix, fait élire 55 de ses candidats, laissant seulement trois sièges à I'opposition, dont un au PCM. 


\section{Les engagements du PCM}

A la différence des campagnes antérieures où le PCM tablait sur des projets d'envergure (construction du métro, Place des Arts, Terre des Hommes, Jeux olympiques, etc.) et sur ses réalisations passées, c'est un programme électoral relativement novateur que ce parti propose en 1986 . On peut expliquer ce changement par l'arrivée du nouveau chef, Claude Dupras, qui tentera de projeter une image de renouveau. Une publicité pré-électorale, publiée par le PCM dans La Presse le 26 août 1986, confirme ce désir de changement et présente Claude Dupras comme uune force nouvelle».

Au total, le PCM rendra publics 45 engagements (Tableau 1), dont la plus grande partie, soit $42 \%$, concerne la mission "aménagement et développement". Viennent ensuite les missions "gouvernementale et administrative» (31\%) et "communautaire" (27\%). II n'est fait mention d'aucun engagement à propos de la mission «travaux publics, transport et environnement", ce qui tranche singulièrement avec les anciens discours électoraux du parti.

Parmi les différents secteurs de politiques, c'est sur le développement économique que portent le plus grand nombre d'engagements annoncés dans le discours électoral du PCM en 1986. Plus précisément, le PCM s'engage à faire de Montréal une cité internationale en $y$ attirant plusieurs organismes a caractère international. II promet aussi l'adoption de diverses mesures pour soutenir I' «entrepreneurship" et créer un climat propice à la venue des investisseurs.

Au chapitre de l'habitation, le PCM déclare vouloir poursuivre l'opération 20000 logements en portant l'objectif a 30000 . En ce qui concerne plus particulièrement le logement social, le PCM s'engage à accorder la priorité à l'aide gouvernementale sous forme d'allocation-logement plutôt qu'à la construction de logements publics. En matière d'urbanisme, il compte créer un fonds de développement qui servira à revitaliser le tissu urbain montréalais. II promet en outre un plan d'aménagement pour le centre-ville, plan auquel la population sera associée. 
Tableau 1 : Engagements électoraux rendus publics au cours de la campagne électorale de 1986 à Montréal

\begin{tabular}{|c|c|c|c|c|}
\hline Partis & \multicolumn{2}{|c|}{ PCM } & \multicolumn{2}{|c|}{$\mathrm{RCM}$} \\
\hline & $N$ & $\%$ & $\mathbf{N}$ & $\%$ \\
\hline $\begin{array}{l}\text { Gouv. at administrative (G) } \\
\text { Rel. intergouvernementales } \\
\text { Finances } \\
\text { Gestion politique } \\
\text { Gestion administrative }\end{array}$ & $\begin{array}{l}2 \\
4 \\
4 \\
4\end{array}$ & $\begin{array}{l}4,4 \\
8,9 \\
8,9 \\
8,9\end{array}$ & $\begin{array}{l}0 \\
3 \\
7 \\
7\end{array}$ & $\begin{array}{r}0 \\
5,9 \\
13,7 \\
13,7\end{array}$ \\
\hline Total & 14 & 31,1 & 17 & 33,3 \\
\hline $\begin{array}{l}\text { Communautaire (C) } \\
\text { Sécurité publique } \\
\text { Santé et services sociaux } \\
\text { Sports et loisirs } \\
\text { Culture }\end{array}$ & $\begin{array}{l}1 \\
4 \\
1 \\
6\end{array}$ & $\begin{array}{r}2,2 \\
8,9 \\
2,2 \\
13,3\end{array}$ & $\begin{array}{l}2 \\
5 \\
3 \\
9\end{array}$ & $\begin{array}{r}3,9 \\
9,8 \\
5,9 \\
17,7\end{array}$ \\
\hline Total & 12 & 26.7 & 19 & 37,3 \\
\hline $\begin{array}{l}\text { Travaux publics et env. (T) } \\
\text { Voirie } \\
\text { Transport } \\
\text { Environnement }\end{array}$ & $\begin{array}{l}0 \\
0 \\
0\end{array}$ & $\begin{array}{l}0 \\
0 \\
0\end{array}$ & $\begin{array}{l}0 \\
3 \\
0\end{array}$ & $\begin{array}{r}0 \\
5,9 \\
0\end{array}$ \\
\hline Total & 0 & 0 & 3 & 5,9 \\
\hline $\begin{array}{l}\text { Aménagement et dév. (A) } \\
\text { Urbanisme } \\
\text { Habitation } \\
\text { Développement économique }\end{array}$ & $\begin{array}{r}5 \\
3 \\
11\end{array}$ & $\begin{array}{r}11,1 \\
6,7 \\
24,4\end{array}$ & $\begin{array}{l}5 \\
1 \\
6\end{array}$ & $\begin{array}{r}9,8 \\
2,0 \\
11,8\end{array}$ \\
\hline Total & 19 & 42,2 & 12 & 23,6 \\
\hline Grand total & 45 & 100 & 51 & 100 \\
\hline
\end{tabular}

Dans le domaine des finances, le PCM annonce son intention de ne pas hausser le taux des taxes pour l'année 1987 et de le diminuer par la suite, proportionnellement à la hausse de l'évaluation. Sur le plan de la gestion politique, le PCM désire que les membres du comité exécutif, tels des ministres, aient la responsabilité de secteurs précis de 
I'administration municipale et répondent de leurs dossiers devant le conseil. Le PCM promet de consulter les citoyens par la tenue de sommets ou l'organisation de débats publics. II se propose également d'instaurer un processus formel de consultation par la création de commissions ad hoc formées de conseillers issus de tous les partis. II s'oppose cependant au référendum en tant que mécanisme de consultation. En matière de gestion administrative, le PCM projette l'ouverture de bureaux de quartier et promet l'adoption d'une politique favorisant, sur la base d'une compétence égale, l'embauche de femmes et de membres des minorités ethniques dans I'administration municipale.

En 1986, le PCM manifeste également un grand intérêt pour la mission communautaire. II s'engage à favoriser la création d'un réseau de refuges pour les femmes victimes de violence et à lutter contre la prostitution et la drogue. II entend favoriser l'implantation de garderies dans les grands projets immobiliers. Dans le domaine de la culture, le PCM veut augmenter la participation des communautés culturelles à la vie montréalaise, organiser la tenue d'un festival multiculturel et accroitre le budget du Conseil des arts de la CUM.

Voilà donc un menu électoral assez différent de ceux qui firent le succès du maire Drapeau. Trois raisons principales contribuent à expliquer ce changement. II y a d'abord les efforts tentés par Claude Dupras pour moderniser et démocratiser le parti et le doter d'un programme. Ses efforts cependant ne feront pas l'unanimité comme le prouvera plus tard l'éclatement au grand jour des luttes de pouvoir au sein du PCM (Leblanc, 1989). II y a, deuxièmement, la présence de plus en plus menaçante du RCM à qui les sondages accordent, depuis déjà plusieurs mois, une forte avance dans les intentions de vote. La troisième raison, enfin, c'est qu'après vingt-six ans de régime Drapeau, des élus et des organisateurs du PCM sont forcés d'admettre la nécessité d'avoir un hôtel de ville plus ouvert aux citoyens et surtout d'être davantage à l'écoute des principaux acteurs socio-économiques locaux.

En fait, ce virage opéré par le PCM s'était amorcé avant l'arrivée de Claude Dupras à la direction du parti soit au début 
des années 80. Le PCM, en grande partie sous l'initiative d'Yvon Lamarre, président du comité exécutif, avait alors adopté une série de mesures (nouveaux programmes à caractère économique et réformes administratives) qui feront apparaître la victoire du RCM en 1986 plutot en continuité qu'en rupture profonde avec les dernières années du régime Drapeau (Léonard et Léveillée, 1986c: $55-81$ et Léveillée, 1988). De ce point de vue, on peut affirmer qu'une bonne partie des engagements faisant partie de la plate-forme électorale du PCM en 1986 sont le reflet de la «nouvelle» gestion politique et administrative mise de l'avant par ce parti entre 1982 et 1986.

\section{Les engagements du RCM}

A plusieurs égards, le discours électoral du RCM au cours de la campagne électorale de 1986 affiche, lui aussi, des nouveautés. Avant d'en faire état, comparons la structure de son discours a celle du PCM (Tableau 1). II ressort, en premier lieu, que le discours du RCM contient un plus grand nombre d'engagements que celui du PCM (51 contre 45). Cette différence apparaît plutôt minime si on considère que le programme officiel du RCM compte 90 pages en 1986, alors que le manifeste politique rendu public par le PCM, au mois d'octobre 1986, en compte à peine une cinquantaine.

En ce qui concerne la répartition des engagements par mission, deux différences importantes sautent aux yeux. La première est la place beaucoup plus grande accordée par le PCM à la mission "aménagement et développement" à laquelle se greffent $42 \%$ de ses engagements, comparativement à un peu moins de $24 \%$ dans le cas du RCM. Cette différence, comme nous l'avons vu, est en grande partie attribuable à l'accent que met le PCM sur le thème du développement économique pour lequel la proportion de ses engagements est deux fois plus élevée $(24 \%)$ que celle des engagements du RCM (12\%).

La deuxième différence concerne la mission communautaire. Le RCM lui consacre $37 \%$ de ses engagements contre $27 \%$ dans le cas du PCM. L'importance 
plus grande accordée par le RCM aux loisirs et à la culture, explique cet écart. En dépit de ces différences, les deux partis accordent la même place, selon un ordre décroissant d'importance, aux missions kgouvernementale et administrative" et "travaux publics et environnement". Dans les deux cas, en effet, ces missions se classent respectivement deuxième et quatrième.

Résumons maintenant les principaux engagements du RCM en fonction de l'importance que ce parti accorde, par ordre décroissant, aux différentes missions municipales.

La plus grande partie des engagements du RCM reliés a la mission communautaire concerne la culture. Le parti parle de créer un service municipal des affaires culturelles, à mettre sur pied un bureau de renseignements a l'intention des minorités ethniques à l'hôtel de ville et à créer un conseil consultatif sur le développement culturel. II promet également la mise sur pied d'un fonds d'investissement mixte, qui servira à doter Montréal des équipements culturels qui lui manquent.

En regard de la mission gouvernementale et administrative, le RCM précise, d'entrée de jeu, que les conseils de quartier ne verront pas le jour au cours d'un premier mandat. II entend cependant créer des commissions permanentes du conseil (ad hoc dans le cas du PCM), accorder une période de questions aux citoyens lors des assemblées du conseil, instaurer une politique d'accès à l'information, et donner aux citoyens la possibilité de recourir au mécanisme du référendum (ce à quoi s'oppose fermement le PCM).

Sur le plan administratif, le RCM déclare vouloir accroitre la mobilité chez les cols bleus, limiter la sous-traitance, instaurer un programme d'action positive a l'égard des femmes et des membres des minorités ethniques et favoriser l'accessibilité aux services municipaux dans les quartiers. En ce qui concerne les finances municipales, il promet l'abolition de la taxe d'eau et une répartition plus équitable du fardeau fiscal entre les différentes catégories de contribuables.

Au chapitre du développement économique, le RCM annonce qu'il va favoriser la relance industrielle dans l'est de Montréal, travailler à l'expansion du Port de Montréal, créer 
une zone franche portuaire et implanter des centres d'incubation d'entreprises. En matière d'aménagement et d'habitation, le RCM entend revoir l'aménagement des quartiers pour favoriser une plus grande intégration des fonctions résidentielle et commerciale. II se dit favorable à une participation plus grande des professionnels du secteur privé a la conception des projets municipaux d'urbanisme. II promet enfin d'imposer un moratoire sur tous les projets de construction sur le Mont-Royal et de créer un réseau d'espaces verts.

Le RCM prend trois positions relativement aux travaux publics, transport et environnement. Deux de ces positions concernent le transport en commun. La première, qui est l'abandon du projet de rendre le transport en commun gratuit pour tous les usagers, représente un net recul. La seconde cependant est la limitation des coûts de ce service : les usagers ne défraieraient qu'un tiers des coûts.

La comparaison des plates-formes électorales du PCM et du RCM au cours de la campagne électorale de 1986 permet de tirer trois conclusions préliminaires. On constate tout d'abord que les deux partis s'opposent surtout en ce qui concerne la mission gouvernementale et administrative. Par exemple, bien que le discours du PCM en 1986 diffère largement de celui que tenait le maire Drapeau, les engagements du parti en matière de démocratie et de gestion administrative demeurent plus généraux et moins interventionnistes que ceux du RCM. Cependant le PCM accorde une plus grande importance aux finances et à la fiscalité municipale, deux thèmes fortement associés au développement économique. En cela, son discours électoral pendant la campagne reflète bien l'objectif prioritaire de son programme, centré sur le développement économique.

En matière économique, pour être réaliste, il faut reconnaitre que tout progrès passe par la prospérité. Le capital est le nerf de la guerre et il permet de réaliser ce qui est souhaitable. Pour le parti civique, l'essor social et culturel passe 
d'abord par le développement économique (PCM, 1986: 22).

Sans nier l'importance du développement économique, le RCM l'associe plutôt à la démocratie locale et à la qualité de vie.

Une administration RCM fera du développement économique ... l'une de ses priorités. Cette priorité se reflétera, entre autres, par la volonté ... d'intégrer les débats économiques a la vie publique et politique municipale, dans un souci d'une plus grande démocratisation de l'activité économique. Convaincu que qualité de vie et développement économique peuvent et doivent aller de pair... (RCM, 1986: 31).

Deuxième constatation : le RCM a tenu, au cours de cette campagne, un discours rassurant et moins dogmatique. Non seulement ses propos ont-ils servi à dédouaner le parti par rapport à certains de ses engagements antérieurs, mais ils lui ont permis aussi d'établir une distinction claire entre son programme de parti et son programme de gouvernement. Le RCM était d'autant plus porté à faire cette distinction que les sondages le donnaient gagnant bien avant le déclenchement de la campagne. Stratégiquement, son discours électoral devait lui permettre de préserver, sinon d'accroître, son avance sans s'adresser exclusivement à ses clientèles traditionnelles.

On note, en troisième lieu, un rapprochement entre les deux formations politiques, et cela malgré des divergences en certains domaines. Le départ de Jean Drapeau et l'arrivée de Claude Dupras à la tête du PCM, I'orientation idéologique moins militante et plus conciliante adoptée par les principaux ténors du RCM au tournant des années 80 (Léonard et Léveillée, 1986cl, la conjoncture économique et, sans doute aussi, le désir de changement chez bon nombre d'électeurs 
montréalais sont au nombre des principales raisons responsables de ce rapprochement. Un rapprochement qui se produira également entre le PCQ et le RPO sur la scène politique municipale de Québec.

\section{La campagne électorale de 1989 à Québec}

C'est à une campagne électorale municipale particulièrement animée et au dénouement imprévu qu'assisteront les électeurs de Québec en 1989. Nombre d'électeurs et d'observateurs s'attendaient pourtant à une élection sans surprise. Les sondages rendus publics avant le déclenchement de la campagne et jusqu'à deux semaines avant les élections indiquaient, en effet, que le Progrès civique de Québec (PCQ), au pouvoir depuis 1965, allait être réélu sans difficulté. Or, $c^{\prime}$ est son adversaire, le Rassemblement populaire de Québec (RPQ), qui va finalement remporter cette élection en recueillant $62 \%$ des voix à la mairie et en faisant élire seize de ses candidats sur vingt et un aux postes de conseillers. Les changements que ces deux partis ont connus sur le plan de leur leadership ne sont pas étrangers à ces résultats.

C'est en octobre 1988 que le RPO se donne un nouveau chef, le troisième depuis sa création en 1977. Seul candidat en lice, Jean-Paul L'Allier, ancien ministre dans le gouvernement libéral de Robert Bourassa de 1970 à 1976, accède alors a la direction du parti. Des changements sont aussitôt amorcés. Le programme officiel du parti est d'abord révisé en profondeur et, pour la première fois, une distinction claire est faite entre le programme officiel et la plate-forme électorale contenant les engagements qui devront être tenus au cours d'un premier mandat (Quesnel et Belley, 1991 : 148).

Du côté du PCQ, l'heure est également aux changements. Sur le plan du "leadership" d'abord : au printemps 1989, Jean Pelletier, chef du parti et maire depuis 1977, annonce qu'il ne sollicitera pas un nouveau mandat. Au mois de mai, soit à peine six mois avant l'élection de novembre 1989, Jean-François Bertrand, ancien ministre péquiste de 1976 a 1985, devient le nouveau chef du PCO. 
- Il accède à la direction du parti après s'être engagé à en démocratiser les structures et a élaborer un programme politique officiel. $A$ la fin de septembre, les membres-délégués du parti, réunis en congrès, adoptent officiellement la nouvelle constitution et le programme conçus par une équipe composée principalement d'amis et de proches collaborateurs de Jean-François Bertrand du temps où il était ministre. Plusieurs wanciens" élus, organisateurs et travailleurs électoraux du PCQ feront ainsi les frais de la "cure de rajeunissement" entreprise par le nouveau chef. II s'ensuivra des frustrations qui mineront le moral des troupes dans plusieurs districts.

C'est donc dans un contexte pré-électoral très différent de celui qui prévalait à Montréal en 1986 que s'est amorcée la lutte à la mairie de Québec à l'automne 1989. Si le contexte général est beaucoup plus favorable au parti au pouvoir à Québec, il y a cependant un élément du débat électoral dont le PCQ n'avait pas prévu toute l'importance. II s'agit du gigantesque projet immobilier de la Grande Place dans la basse-ville qui avait reçu l'aval de l'administration sortante au mois d'août 1989 et que le nouveau chef du PCO, après quelques hésitations, appuiera finalement sans réserve. Le RPQ, fortement opposé a ce projet, en fera le principal enjeu de la campagne, le présentant dans son discours comme le symbole d'une administration vouée aux intérêts des gros promoteurs privés.

\section{Les engagements du PCO}

Au total, le PCQ rendra publics 41 engagements au cours de cette campagne (Tableau 2). C'est la mission "gouvernementale et administrative" qui en absorbe le plus grand nombre (39\%) suivie des missions «travaux publics et environnement" ( $32 \%)$, "aménagement et développement" $(24 \%)$ et, loin derrière, de la mission "communautaire» (5\%).

La priorité accordée à la gestion politique et à l'urbanisme découle de la promesse faite par Jean-François Bertrand de faire participer les citoyens à la gouverne municipale. Ainsi, pour rapprocher l'administration municipale 
Tableau 2 : Engagements électoraux rendus publics au cours de la campagne électorale de 1989 a Québec

\begin{tabular}{|l||r|r|r|r|}
\hline \multicolumn{2}{|c|}{ Partis } & \multicolumn{2}{c|}{ PCO } & \multicolumn{2}{c|}{ RPO } \\
\hline Missions & $N$ & $\%$ & $N$ & $\%$ \\
\cline { 2 - 5 } Gouv. ot administrative (G) & & & & \\
Rel. intergouvernementales & 2 & 4,9 & 2 & 3,3 \\
Finances & 4 & 9,8 & 3 & 5,0 \\
Gestion politique & 7 & 17,1 & 8 & 13,3 \\
Gestion administrative & 3 & 7,3 & 3 & 5,0 \\
\hline Total & 16 & 39,1 & 16 & 26,7 \\
\hline Communautaire (C) & & & & \\
Sécurité publique & 0 & 0 & 3 & 5,0 \\
Santé et services sociaux & 0 & 0 & 0 & 0 \\
Sports et loisirs & 2 & 4,9 & 4 & 6,7 \\
Culture & 0 & 0 & 6 & 10,0 \\
\hline Total & 2 & 4,9 & 13 & 21,7 \\
\hline Travaux publics et env. (T) & & & & \\
Voirie & 6 & 14,6 & 1 & 1,7 \\
Transport & 6 & 14,6 & 5 & 8,3 \\
Environnement & 1 & 2,4 & 3 & 5,0 \\
\hline Total & 13 & 31,7 & 9 & 15,0 \\
\hline Aménagement et dév. (A) & & & & \\
Urbanisme & 7 & 17,1 & 14 & 23,3 \\
Habitation & 0 & 0 & 4 & 6,7 \\
Développement économique & 3 & 7,3 & 4 & 6,7 \\
\hline Total & 10 & 24,4 & 22 & 36,7 \\
\hline & & & & \\
\hline Grand total & 41 & 100 & 60 & 100 \\
\hline
\end{tabular}

de la population, le chef du PCQ promet d'organiser une rencontre annuelle dans chacun des districts de la ville et de tenir les séances du conseil municipal en début de soirée plutôt qu'en fin d'après-midi. II prend aussi la résolution d'étudier la possibilité d'implanter des bureaux de 
renseignements dans chacun des quatre grands secteurs de la ville.

Sur le plan administratif, le PCO s'engage à réduire par attrition le nombre de fonctionnaires et a recourir plus fréquemment a la sous-traitance lorsque cela pourra être avantageux pour la ville. Des engagements qui provoqueront immédiatement des réactions négatives de la part des différents syndicats représentant les employés municipaux. La prudence du RPQ sur cette question lui méritera au contraire leur appui.

Sur le plan des finances municipales, le chef du PCO promet de faire des économies de $25 \mathrm{M} \$$ au cours du prochain mandat en cherchant notamment à contenir l'augmentation des dépenses au-dessous du taux d'inflation. II veut éliminer complètement, au cours des cinq prochaines années, l'écart fiscal entre la ville de Québec et les villes de la banlieue. Les nombreux engagements du PCQ en matière de travaux publics sembleront cependant contredire quelque peu l'objectif qu'il s'était fixé de réduire les dépenses.

Au total, ce sont quelque $12 \mathrm{M} \$$ que le PCO s'engage a dépenser au cours des quatre prochaines années pour la construction et l'amélioration des infrastructures dans les différents quartiers de la ville et l'aménagement de pistes cyclables. Au chapitre de l'environnement, le PCQ s'engage à consacrer $2 \mathrm{M} \$$ à la mise sur pied d'un programme de collecte sélective et de recyclage des déchets.

À la mission "aménagement et développement", la troisième en importance dans le discours du PCQ, est relié l'engagement de créer un comité de vigilance qui veillera à ce que la construction du complexe immobilier de la Grande Place se fasse dans le respect de l'environnement et de la qualité de vie des résidents du quartier. "Dorénavant, affirme le chef du $\mathrm{PCO}$, en matière d'aménagement, les intérêts des résidents vont passer avant ceux des bâtisseurs et des promoteurs.... (Collard, 1989). En matière de développement économique, le $\mathrm{PCO}$ promet de consacrer au cours des quatre prochaines années $6,5 \mathrm{M} \$$ au développement de la vocation commerciale de quelques rues.

En ce qui concerne la mission communautaire, les engagements du PCQ se limitent à des investissements de 
quelque $2 \mathrm{M} \$$ pour la construction de nouveaux parcs a travers la ville.

Comme le PCM en 1986, le PCQ fait donc en 1989 une plus grande place a la consultation des citoyens et cherche a prendre ses distances («dorénavant») avec les politiques antérieures du parti en matière d'aménagement et de développement. Les préoccupations traditionnelles du PCO a l'égard de la fiscalité et des travaux publics n'en continuent pas moins cependant à occuper une place importante dans son discours.

\section{Les engagements du RPO}

Le nombre des engagements pris par les deux partis est très inégal : le RPO domine avec 60 engagements contre 41. La structure de leurs discours diffère également sur trois points (Tableau 2). D'abord en ce qui concerne la mission communautaire, les engagements du RPQ sont six fois plus nombreux que ceux du PCO. L'importance accordée par le RPO à la culture explique en grande partie cet écart. Ensuite, en rapport avec la mission "aménagement et développement", l'urbanisme et I'habitation accaparent $30 \%$ des engagements du RPQ contre $17 \%$ seulement dans le cas du PCO. Enfin, le secteur de la voirie regroupe $15 \%$ des engagements du PCO contre $2 \%$ seulement de ceux du RPO. Le classement des missions, suivant un ordre décroissant, est donc A-G-C-T dans le cas du RPQ et G-T-A-C dans celui du PCQ. La structuration différente des discours partisans et, conséquemment, du débat électoral à Québec comparativement à Montréal, explique que la définition des enjeux ne soit pas tout à fait la même dans les deux villes. Nous reviendrons sur ce point un peu plus loin; résumons auparavant les principaux engagements du RPO en commençant par la mission gouvernementale et administrative.

Pour mettre fin au favoritisme et aux conflits d'intérêts dont se seraient rendus coupables certains membres de I'administration sortante, le RPO promet d'adopter un code de déontologie auquel seront soumis tous les élus. De plus, if entend rendre possible pour les citoyens le recours à la 
procédure du référendum relativement aux projets de modification du zonage.

En ce qui concerne la représentation politique des femmes, le RPO s'engage à nommer deux femmes au Comité exécutif et à appliquer la règle de la représentation égale dans tous les comités et commissions relevant du conseil municipal. II veut aussi mettre sur pied un programme d'accès à l'égalité et entend appliquer une politique d'équité salariale dans la fonction publique.

Dénonçant les coupures de personnel envisagées par le PCO, le RPO s'engage a revoir la structure administrative et à évaluer la pertinence et l'efficacité de certains services municipaux. En matière de finances, il se propose de maintenir la hausse annuelle moyenne du compte de taxe à $1,5 \%$ au-dessous du taux d'inflation. II compte y arriver en incitant les nouveaux ménages à venir s'installer à Québec et en réduisant les dépenses d'immobilisation.

Comme le RCM en 1986, le RPO ne prend aucun engagement au cours de la campagne électorale sur la question, pourtant centrale dans son programme, des conseils de quartier. Le chef du parti en fera mention une seule fois, à la fin août, soit une dizaine de jours avant le début officiel de la campagne électorale, en disant que "l'établissement des conseils de quartier ne sera jamais imposé... on en fera la promotion et si la population n'en veut pas, elle n'en aura pas" (Routhier, 1989a).

En matière d'urbanisme, le RPQ promet de ne pas donner suite au projet de la Grande Place. II propose à la place un projet aux dimensions plus réduites, axé davantage sur la fonction résidentielle et dont la réalisation sera précédée de consultations publiques. Dans le domaine de l'habitation, omis par le PCQ, le RPO a l'intention de construire des logements coopératifs, de bonifier les programmes provinciaux d'accès a la propriété et de favoriser la rénovation des immeubles locatifs. Pour aider le développement économique, le parti promet la mise sur pied de programmes favorisant la création d'emplois dans la basse-ville, la revitalisation des artères commerciales et le développement des petites et moyennes entreprises. 
C'est cependant à l'égard de la mission communautaire, et plus précisément de la culture, que le discours électoral du RPO se différencie quantitativement le plus de celui du PCQ. Déclarant que la "culture doit être à Québec ce que I'automobile est à Détroit» (Routhier, 1989b), le chef du RPO promet d'augmenter de $3 \mathrm{M} \$$ (de la date des élections a 1994) le soutien financier de la ville aux professionnels de la culture. La création, au niveau régional, d'un Conseil des arts et de la culture de même que l'implantation, à Québec, de plusieurs institutions culturelles d'envergure nationale et internationale font partie des promesses. Dans le domaine des loisirs, le RPO parle de construire des centres communautaires et d'améliorer les parcs existants.

En travaux publics, transport et environnement, les engagements du RPO concernent surtout le transport en commun. Le RPQ réitère d'abord son intention d'accorder aux personnes âgées le transport gratuit en tout temps a bord des autobus de la Commission de transport de la Communauté urbaine de Québec. II s'engage ensuite à conduire une étude sur les besoins en matière de transport et de circulation avant de donner son aval à la construction d'un métro de surface pour desservir le projet controversé de la Grande Place.

Une grande partie du discours électoral diffusé par le RPO pendant la campagne a donc été centrée sur l'enjeu de la Grande Place. Dans ses engagements en matière de démocratie, de finances, de transport ou d'aménagement, il fait référence directement ou indirectement à ce vaste projet immobilier. L'important traitement médiatique du projet encouragé en cela par les accusations répétées lancées par le chef du RPO, obligera le nouveau chef du PCQ, à maintes reprises pendant la campagne, à se porter publiquement à sa défense. Un peu décontenancée par la tournure de la campagne, l'organisation du PCQ $n$ 'arrivera pas à relancer le débat électoral dans une direction plus favorable. De plus, pour la première fois, la redoutable machine électorale du PCO accusera quelques ratés sur le terrain : plusieurs anciens travailleurs d'élection digéreront mal d'avoir été "tassés" par la "gang à Bertrand» et manifesteront peu d'enthousiasme. La décevante performance de Jean-François Bertrand lors du débat télévisé, qui a eu lieu moins de deux semaines avant le 
scrutin et où le projet immobilier de la Grande Place a encore été longuement débattu, a marqué un point de non-retour pour le $\mathrm{PCO}$.

\section{Les enjeux mobilisateurs du changement}

Nous voudrions terminer cette analyse en comparant les deux élections à trois points de vue : le wleadership" au sein des partis, le rôle des campagnes électorales et les principaux enjeux électoraux.

II est clair que les changements a la tête des partis civiques ont été a la fois plus difficiles a concilier sur le plan idéologique et plus difficiles à «gérer" sur le plan électoral. La période de transition, d'à peine six mois, laissée aux nouveaux chefs a été trop courte, tant les changements souhaités et entrepris ont bouleversé les anciennes façons de penser et de faire. Le renouvellement du "leadership» survenu plus tôt au sein des partis populaires a renforcé, au contraire, la cohésion et la mobilisation d'autant plus fortes qu'à Montréal la victoire apparaissait à portée de main, et qu'à Québec, l'élection de 1989 était considérée, par plusieurs militants de la première heure, comme celle de la «derniere chance" (Quesnel et Belley, 1989: 77). Cela explique aussi le caractère plus "électoraliste" des discours électoraux.

Les partis populaires se sont en effet appliqués, au cours des deux élections, a promouvoir un programme de "bon gouvernement". Ils ont pris soin, par exemple, de différer ou d'abandonner la réalisation de certains engagements plus risqués comme les conseils de quartier et la gratuité du transport en commun pour tous les usagers. Ils ont également abordé des thèmes au "goût du jour" : la rationalisation administrative, la représentation des femmes et la culture. Un rapprochement idéologique ne pouvait ainsi manquer de se produire entre ces partis et les partis civiques. Mais il subsiste encore des différences marquées entre ces deux familles politiques sur le plan des valeurs. Chez les partis civiques, on note une préférence pour les valeurs plus matérialistes comme le développement économique, la fiscalité et les travaux publics. Chez les partis populaires, on 
observe une préférence pour les valeurs moins matérialistes comme la démocratie, l'égalité sexuelle et la qualité de vie.

Quant aux campagnes électorales elles-mêmes, il ne fait pas de doute que celle de 1989 à Québec a eu sur le vote un effet plus déterminant que celle de 1986 a Montréal. La campagne de Montréal est venue confirmer, en même temps qu'amplifier, une tendance qui était favorable au RCM depuis plusieurs mois. A Québec, la campagne est venue, au contraire, renverser une tendance qui, dès le printemps 1989, favorisait largement le PCQ. Le rôle des médias a été l'un des facteurs responsables de ce retournement important de la conjoncture politique à Québec. Leur insistance et leurs positions sur certains enjeux comme la Grande Place, ou certains événements clés comme le débat télévisé des chefs ont certainement eu une influence sur l'opinion des électeurs. On ne peut cependant passer sous silence les stratégies discursives que les acteurs partisans ont déployées pour essayer de tourner le débat électoral à leur avantage. Sans aucun doute, la personnalité et les talents de communicateurs des chefs ont été pour quelque chose dans les résultats électoraux, tant il est vrai qu'en période électorale «les partis n'existent que relationnellement" (Offerle, 1987: 91). II apparaît donc que les partis populaires et leurs chefs ont été, cette fois-ci, plus habiles que leurs rivaux pour imposer leurs thèmes et définir les enjeux.

Le Tableau 3 montre, de ce point de vue, que la gestion politique et l'urbanisme ont été, dans les deux villes, les enjeux électoraux les plus importants en 1986 et en 1989. On peut penser que la forte structuration du débat électoral autour de ces deux enjeux a eu d'autant plus d'effets sur le vote que les partis populaires ont constamment cherché à les relier. Les partis populaires se sont employés, en effet, à convaincre les électeurs que l'aménagement de la ville, sous le règne des partis civiques, serait inséparable du type de gestion politique propre a ces formations politiques. Lorsqu'en 1986 et 1989 , les nouveaux leaders des partis civiques insisteront sur la nécessité de faire une plus grande place a l'information et à la consultation, ils se trouveront du même coup à lutter, pour la première fois, sur le même terrain que leurs adversaires. Les partis civiques reconnaîtront 
Tableau 3 : Importance accordée par les partis aux différents secteurs de politiques dans leurs plates-formes electorales *

Elections de 1986 à Montréal et de 1989 à Québec

\begin{tabular}{|l|c|c|c|c|}
\cline { 2 - 5 } & \multicolumn{2}{c|}{ Montréal } & \multicolumn{2}{c|}{ Québec } \\
\hline & PCM & RCM & PCO & RPO \\
Relations & & & & \\
intergouvernementales & - & - & - & - \\
Finances & \pm & - & \pm & - \\
Gestion politique & \pm & \pm & \pm & \pm \\
Gestion administrative & - & \pm & - & - \\
\hline Sécurité publique & - & - & - & - \\
Santé et services sociaux & - & \pm & - & - \\
Sports et loisirs & - & - & - & - \\
Culture & \pm & \pm & - & \pm \\
\hline Travaux publics & - & - & \pm & - \\
Transport & - & - & \pm & - \\
Environnement & - & - & - & - \\
\hline Urbanisme & \pm & \pm & \pm & + \\
Habitation & - & - & - & - \\
Développement & + & \pm & - & - \\
économique & & \pm & & \\
\hline
\end{tabular}

- + :lorsque le secteur regroupe plus de $20 \%$ du total des engagements

\pm :lorsque le secteur regroupe entre 10 et $20 \%$ du total des engagements

- : lorsque le secteur regroupe moins de $10 \%$ du total des engagements

implicitement le bien-fondé des positions et dénonciations «traditionnelles" des partis populaires à l'égard de la démocratie locale.

A Québec et à Montréal, lors des élections de 1986 et de 1989, y a-t-il eu d'autres enjeux mobilisateurs que la gestion politique et l'urbanisme? L'importance accordée par les partis aux différents secteurs de politiques en définit-elle d'autres? Le Tableau 3 indique que ce serait le cas du 
développement économique et de la culture à Montréal, deux secteurs auxquels le PCM et le RCM accordent une importance moyenne. A Québec, la définition des autres enjeux est moins claire : ce qui est moyennement important pour un parti ne l'est pas pour l'autre. C'est que la structuration des discours partisans est plus différencielle a Québec qu'à Montréal. Par ailleurs, les relations intergouvernementales, la sécurité publique, les sports, les loisirs et, de façon plus surprenante, l'environnement et I'habitation n'ont pas constitué de véritables enjeux pour les quatre partis au moment des élections de 1986 et de 1989.

\section{Conclusion}

L'analyse des plates-formes électorales des partis municipaux de Montréal et de Québec, lors des élections de 1986 et de 1989, montre que, douze ans après la mise en place d'un véritable système partisan dans ces villes, des divergences idéologiques profondes séparent encore les partis civiques et les partis populaires. Toutefois, les changements survenus dans l'environnement du système politique de ces villes depuis une trentaine d'années, la dynamique politique interne au système partisan et, plus récemment, le renouvellement du "leadership" dans les partis ont contribué a les rapprocher sur certaines questions. Les partis de la première génération, dits "civiques», ont été forcés de faire une place plus grande dans leur discours à la démocratie locale; les partis de la deuxième génération, dits "populaires", ont dû, dans une conjoncture économique plus difficile, adoucir, voire abandonner, les engagements les plus "socialisants" de leur programme.

Si la comparaison des plates-formes électorales montre que les partis de même famille politique $n^{\prime}$ ont pas fait campagne sur la base d'un discours dont la structure et les grandes orientations sont en tous points les mêmes, on peut cependant affirmer que le renversement des partis civiques par les partis populaires s'est fait sur deux enjeux communs fortement mobilisateurs : la démocratie et l'aménagement. De ce point de vue, le RCM et le RPO ont réussi à porter ces 
thèmes jusqu'à ce qu'ils deviennent des enjeux stratégiques décisifs dans le débat électoral.

Depuis l'arrivée au pouvoir du RCM à Montréal et du RPO a Québec, sont survenus des changements qui concernent ces partis, les "nouveaux" partis d'opposition de même que le système partisan dans ces villes. Si la démission, en 1989, de quatre conseillers du RCM n'a pas empêché ce parti d'être rélu en novembre 1990, elle a cependant conduit à la création d'un nouveau parti municipal plus à gauche, la Coalition démocratique de Montréal. Un autre parti, le Parti municipal de Montréal, qui se situe idéologiquement à droite du RCM, est né des nombreux problèmes internes qu'éprouve le PCM depuis sa défaite cuisante en 1986. À l'occasion de l'élection municipale de 1990 , les "verts» ont fait, eux aussi, leur entrée officielle sur la scène politique en créant leur parti : le Montréal écologique. Quant au PCM, son avenir semble de plus en plus incertain depuis le départ de Claude Dupras en janvier 1989 et la démission de son successeur, Nicole $G$. Larocque en mars 1991. Après quelques années de bipartisme, la ville de Montréal est donc revenue à une situation de multipartisme. A Québec, le bipartisme s'est maintenu malgré les tensions internes du RPQ, tensions qui ont amené le départ, au printemps 1991, d'un conseiller qui siège depuis comme indépendant. Du côté du PCO, la défaite de 1989 a entraîné la démission de Jean-François Bertrand. C'est un conseiller, réélu en 1989, qui assume depuis par intérim la direction du parti. Un nouveau chef devrait être élu en 1992.

La réorganisation actuelle des forces politiques partisanes dans les villes de Montréal et de Québec, montre bien que la transformation des partis et des systèmes partisans ne s'arrête jamais. La source des transformations actuelles se situe cependant bien plus à l'intérieur qu'à l'extérieur du système politique municipal. Elles se réalisent en dépit de la fixité relative des institutions municipales qui modelent le «jeu» des acteurs partisans.

\footnotetext{
- L'auteur tient à remercier le Conseil de recherche en sciences humaines du Canada pour l'aide financière qu'il a accordée à la réalisation de cette recherche. La première version de ce texte a fait l'objet d'une communication présentée dans le cadre du congrès de I'ACFAS (section
} 
*Science politiquex) tenu a l'Université de Sherbrooke au mois de mai 1991. L'auteur remercie M. Pierre-Yves Melançon pour les commentaires qu'il a faits a cette occasion. Ses remerciements vont également à Madame Louise Quesnel de l'Université Laval qui a bien voulu lire et commenter la première version de ce texte. 
Bibliographie

ANDREW, C., J. LÉVEILLÉE et Louise QUESNEL, "Le pouvoir local et les stratégies de réalignement dans les centres urbains du Québecn, Anthropologie et sociétés, vol. 9, $n^{\circ} 2,1985$, p. 117-149.

BEAUREGARD, L., kLes élections municipales de Montréal (novembre 1978)", dans BOURASSA, G. et J. LÉVEILLEE (sous la direction de), Le système politique de Montréal, Montréal, ACFAS, (Les cahiers de I'ACFAS n० 431, 1986, p. 373-392.

BERNARD, F., kAvec Roger D. Landry, le Parti civique serait encore au pouvoir: Nicole Gagnon Larocque», La Presse, $1^{*}$ novembre 1990, p. A-18.

COLLARD, M. "1000 personnes au "show" du Progrès civique. J.-F. Bertrand répète que ça va changer», Le Soleil, 26 octobre 1989, p. A-3.

COLLIN, J.-P., "La régionalisation des forces politiques contemporaines dans la ville de Montréal. Regards sur les élections municipales depuis 1970", dans BOURASSA, G. et J. LÉVEILLÉE (sous la direction de), Le système politique de Montréal, Montréal, ACFAS (Les cahiers de l'ACFAS $n^{\circ} 43$ ), 1986, p. 353-372.

EZOP-QUÉBEC, Une ville à vendre, Laval, Éditions coopératives Albert Saint-Martin, 1981.

GAGNON-LACASSE, F. et G. BOURASSA, «Le vote à la mairie aux élections du 14 novembre 1982 : de nouvelles tendances», dans BOURASSA, G. et J. LÉVEILLEEE (sous la direction de), Le système politique de Montréal, Montréal, ACFAS (Les cahiers de I'ACFAS $n^{\circ} 43$ ), 1986, p. 393-404.

GERSTLE, J. La communication politique, Paris, PUF, IColl. Que sais-je? $n^{\circ} 26521,1992$. 
KAPLAN, H., Reform, Planning and City Politics : Montréal, Winnipeg, Toronto, Toronto, University of Toronto Press, 1982.

LEBLANC, G., "Les enfants récupèrent l'héritage», La Presse, 15 mai 1989 , p. B-3.

LEMIEUX, V., Systèmes partisans et partis politiques, Sillery, P.U.Q., 1985.

LÉONARD, J.-F. et J. LÉVEILLÉE, (1986a), "Le RCM comme opposition: de la reconnaissance à la crédibilité», Le Devoir, 30 octobre 1986.

LÉONARD, J.-F. et J. LÉVEILLÉE, Montréal after Drapeau, Montréal, Black Rose Books, $1986 \mathrm{~b}$.

LÉONARD, J.-F. et J. LÉVEILLÉE, (1986c), "Qui gouvernera à l'hôtel de ville?», Le Devoir, 19 septembre, p. 12.

LÉVEILLEEE, J., "Les stratégies de réponse à la crise de croissance des années soixante-dix à Montréal», dans BOURASSA, G. et J. LÉVEILLÉE (sous la direction de), Le système politique de Montréal, Montréal, ACFAS (Les cahiers de l'ACFAS $n^{\circ} 43$ ), 1986, p. 467-480.

LÉVEILLÉE, J. «Pouvoir local et politiques publiques a Montréal: renouveau dans les modalités d'exercice du pouvoir urbain", Cahiers de recherche sociologique, vol. $6, n^{\circ} 2,1988$. p. 37-63.

MONIĖRE, D. Le discours électoral. Les politiciens sont-ils fiables?, Montréal, Québec/Amérique, 1988.

OFFERLE, M., Les partis politiques, Paris, PUF, IColl. Que sais-je? $\left.n^{\circ} 2376\right), 1987$.

PARTI CIVIQUE DE MONTREAL, Une force nouvelle. Le manifeste du Parti civique de Montréal, 1986.

PELLETIER, R., Partis politiques et société québécoise. De Duplessis a Bourassa, 1944-1970, Montréal, Québec/Amérique, 1989.

QUESNEL-OUELLET, L., "Un parti politique municipal: le Progrès civique de Québec", dans PELLETIER, R. (sous la direction de), Partis politiques au Québec, Montréal, Hurtubise HMH, Ltée, 1976, p. 273-299.

QUESNEL-OUELLET, L. "Aménagement urbain et autonomie locale», dans BERGERON, G. et R. PELLETIER (sous la direction de), L'État du Québec en devenir, Montréal, Boréal Express, 1980, p. 211-238. 
QUESNEL-OUELLET, L., "Les partis politiques locaux au Québec" dans LEMIEUX, V. (sous la direction de), Personnel et partis politiques au Québec, Montréal, Boréal Express, 1982, p. 277-307.

QUESNEL, L. et S. BELLEY, Partis politiques municipaux: une étude de sociologie électorale, Montréal, Les éditions Agence d'Arc, 1991.

RASSEMBLEMENT DES CITOYENS ET DES CITOYENNES DE MONTRÉAL, Programme, 1986.

ROUTHIER, B., «Formation d'un conseil de quartier: le RP favoriserait Saint-Jean-Baptiste», Le Soleil, 1 er septembre, 1989a, p. A-3.

ROUTHIER, B., "L'Allier s'engage à soutenir la culture», Le Soleil, 28 octobre, 1989 b, p. A-3.

SANCTON, A., "Montreal" in MAGNUSSON, W. and $A$. SANCTON, eds, City Politics in Canada, Toronto, University of Toronto Press, 1983, p. 58-93. 